\title{
Experimental and numerical simulation of dough kneading in filled geometries
}

\author{
D.M. Binding, M.A. Couch, K.S. Sujatha and M.F.Webster
}

JFE

\begin{abstract}
The main objective of this study is to counterpart numerical model and experimental studies for rotating flows associated with dough kneading, and validate the flow patterns generated. The flows considered are in a complex domain setting. Two types of cylindrical vessels are studied at various rotational speeds; one with one stirrer and a second with two. Laser Doppler Anemometry is used to obtain the velocity vectors associated with the flow fields. Close agreement is obtained between numerical and experimental flow fields and the magnitudes of velocity vectors. Both sets of results show maximum shear-rates outside the stirring rods. The rate-of-work done also peaks in this region and this is an important quantity to dictate optimal mixer design.
\end{abstract}

\section{Introduction}

This paper is part of a broader study aimed at improving understanding of the technology involved in dough mixing that arises in the food processing industry. Some introductory articles on the subject have already been published (Couch and Binding, 2000, Ding and Webster 2000). This is the first to combine the two strands of numerical simulation and experimental research. Here we study the flows associated with filled geometries, which are simplified scaled-down versions of commercial mixers. The work as a whole includes other issues, such as the development of the free surface in partially-filled vessels and wetting and peeling on the solid/fluid interface. These subjects are to be addressed in further publications. The overall purpose of the study is to be able to optimise mixer design, in terms of energy efficiency and the amount and type of work done on the dough, through a more informed understanding of the process.

The geometries and model fluids investigated in this paper represent intermediate steps in the development of numerical simulations of the types of flow that are encountered in dough kneading in industrial mixers. For industrial mixers, the dough (a complex viscoelastic fluid), which partially fills the geometry, is driven around a bowl by stirring rods that rotate concentrically about the axis of the vessel (See figure 0 for sample pictures of the model 
mixer, under operation and a baked product as a result). However, the two main geometries considered here consist of a cylindrical rotating vessel, fitted with a fixed lid with either one or two stirring rods attached eccentrically with respect to the axis of the vessel. The actual configuration of the two geometries is described in the following section 2 . When these geometries are fully-filled with simple (non-viscoelastic) model fluids the resulting flows, are both three-dimensional in nature and are steady. The fact that the flows are steady, greatly simplifies the task of modeling since it avoids the complication arising from moving the stirrers through the mesh. A more complex and realistic case is also considered where the vessel and lid are held fixed and the rods and base rotate about the centre of the vessel. This case is included in order to demonstrate the progression of the modeling.

The simulation procedure addresses the numerical solution of the fully three-dimensional Generalised Navier-Stokes equations for incompressible flows. This involves a so-called Taylor-Galerkin finite element formulation, which applies a temporal discretisation in a Taylor series prior to a Galerkin spatial discretisation. A semi-implicit treatment for diffusion is employed to address linear stability constraints. The flow is modelled as incompressible via a pressure-correction scheme and an inelastic model with shear rate dependent viscosity is incorporated. Details of the analysis are documented elsewhere (Hawken et al., 1990, Ding et al., 1995 and Matallah et al., 1998).

The experimental component of the work consists of measurements of the flows associated with the geometries and model fluids to validate the numerical simulations in terms of stream function and velocity vectors. In order to record the streamlines associated with the geometries we appeal to the traditional laser scatter technique. The technique has been well documented in the literature; see for example Cochrane et al. (1981), Boger (1982) and Evans and Walters (1985). Here, a suitable model fluid is seeded with tracer particles and illuminated with a plane of laser light, which cuts across the axis of rotation. In this study we shall confine our attention to a Newtonian and a shear-thinning (relatively inelastic) model fluid. It is assumed that, provided the flow is steady, the tracer particles move along the streamlines of the fluid. The paths of the particles, and hence the streamlines, are then recorded with time-lapse photography, using a conventional stills camera.

To enhance these experiments, further analysis of the tracer particle motion is carried out with a digital high-speed motion analyzer system in order to determine the velocity vectors associated with the streamlines. 
For the case of the more advanced geometry, where the stirring rods rotate, Laser Doppler Anemometry is employed to determine the velocity vectors associated with this transient flow.

\section{Experimental Considerations}

\subsection{Rheological characterisation}

The first phase of the experimental work involves the rheological characterisation of a simplified industrial dough, consisting of flour, salt and water $(62 \%, 0.5 \%$ and $37.5 \%$ respectively), in terms of shear, elongation and dynamic properties. Candidate model fluids are characterised alongside the dough in order to develop a fluid with similar rheological properties. However, a viable model fluid is also required to be translucent to facilitate flow visualization and for this reason dilute polymer solutions are employed. Once suitable model fluids have been developed, a subsequent phase of the experimental work is to conduct flow visualization in the model geometries accordingly.

\subsection{Flow visualization}

Figure 1 shows a schematic outline of the model geometry for the one-rod case. The rotating base consists of a horizontal turntable provided by a prototype dough mixer supplied by RHM technology (see Acknowledgements), which is capable of producing rotational speeds in the range of 25 to $450 \mathrm{rpm}$. A Perspex cylinder of internal height $0.14 \mathrm{~m}$ and diameter $0.08 \mathrm{~m}$ is carefully attached to the base, in such a way as to ensure that it is reasonably concentric about the vertical axis of rotation. Any pronounced eccentricity would lead to distortion of the laser scatter images. The cylinder is filled with a transparent model fluid, seeded with particles of polystyrene powder at a concentration of approximately $0.01 \%$ wt/wt. A Perspex lid, fitted with a rigid Perspex rod, is fixed above the cylinder, so that it

makes light contact with the cylinder but does not inhibit its rotational motion. The rod, of diameter $0.009 \mathrm{~m}$, is designed to terminate just above the bottom of the cylinder and is a distance of $0.009 \mathrm{~m}$ from the cylinder wall. The dashed line indicates the position of the second rod, opposite the first, in the two-rod geometry. A horizontal plane of laser light is generated by a laser diode and beam-splitter arrangement, that cuts the cylinder at a height approximately three fifths from the base. To increase the strength of the illumination and to remove shadows a second plane of laser light is projected at right angles to the first. The transparent Perspex lid allows polystyrene particles, illuminated in the laser plane, to be 
visualized from directly above the geometry. Note that, for most of this study only the horizontal plane indicated in figure 4 , and denoted by the dashed line labelled $z 3$, will be considered. However, since the flow field within the geometry is three-dimensional in nature, then data for additional slices (z1, z2 \{comment : no z2-plane vortex-centre data from experiment is provided ?? \} and z4) are also obtained to investigate how the flow patterns vary with depth.

The Reynolds number associated with the geometry can be defined as, $\operatorname{Re}=\rho L V / \mu$ where $\mathrm{L}$ is the gap between the rod and wall, $\mathrm{V}$ the tangential velocity of the cylinder wall, $\mu$ the viscosity and $\rho$ is the model fluid density. For the model fluids under consideration drive speeds of 25, 50, 100 and $200 \mathrm{rpm}$ are applied, which correspond to Reynolds numbers of 2, 4, 8 and 16, respectively.

To obtain laser speckle photographs the motion of the circulating tracer particles is recorded in complete darkness, with a Stills camera, via prolonged exposure times. No analysis is required of the stills photography, since the streaklines are simply traced onto the photographs due to the motion of the bright particles along the streamlines that takes place while the camera shutter is open.

A high-speed digital camera system a Kodak Ektapro HS can also be used, with suitable illumination, to obtain moving images of the tracer particles. However unlike the Stills case, these digital high-speed camera pictures require interpretation to obtain streamlines and velocity vector plots. To achieve this a sequence of digital camera pictures, of a model fluid stirring at a fixed rotation speed, are stacked in a slide show format, see Figure 5. These pictures are scanned through and several evenly spaced particles are chosen and traced, as they change position from picture to picture which corresponds to their motion along the streamlines, until they complete a full circuit. The coordinates ( $\mathrm{x}$ and $\mathrm{y}$ ) of these particles are recorded from each picture using gridlines. Since the frame speed of the camera is fixed at 60fps, the velocities of the particles can be estimated from their motion i.e., through their change of coordinates between successive frames.

Note that, the technique of determining the velocity vectors associated with the streamlines, by analysing the high-speed camera images of the motion of the particles, was developed in-house (rather than purchasing Particle Imaging Velocimetry software). This satisfied the need to track particles throughout their entire cycles so that steady, fullydeveloped conditions could be verified. 


\subsection{Laser Doppler Anemometry}

As stated in section 1, for the more advanced geometry, where the stirring rods are allowed to rotate and the outer vessel is fixed, the flow is time-dependent. Therefore, laser scatter techniques cannot be applied to determine the associated flow field. However, it is common for laser Doppler Anemometers to be equipped with an encoder. In our particular case, this allows for the relative motion of the rods to be subtracted out of the measured velocity field, making the determination of the velocity vectors of this flow possible. The Anemometer used (a two-dimensional argon-ion instrument) is able to measure the velocities of small mica particles seeded in the optically clear model fluid. This is achieved via the Doppler effect, at a precise point in the fluid where the projected laser beams intersect. This velocity vector is resolved from two orthogonal components recorded by two pairs of intersecting beams at right angles to one another. By moving a robotic traverse on which the laser is mounted, the intersection point can be made to move to evenly spaced points along the radius of the geometry at a fixed depth. The rotating rods are then synchronised with the encoder, which enables velocity vectors to be measured at each point on a predetermined axial grid centred on the axis of the geometry. It must be emphasised that the measurement is made through the flat Perspex lid of the geometry and so no correction is needed to cope with refraction, which can occur with curved geometry walls, see for example Bicen (1982).

\section{Numerical Simulation}

Complete details of the analysis are given elsewhere (Hawken et al., 1990, Ding et al.]), so that here only a summary of the basic equations and the problem specification are included for convenience.

\subsection{Basic Equations}

There is a requirement to accommodate an inelastic fluid presentation and as such the viscosity $\mu$ is represented via a Carreau-Yasuda model

$$
\mu=\frac{\mu_{\mathrm{o}}-\mu_{\infty}}{1+(\lambda \dot{\gamma})^{\mathrm{m}}}+\mu_{\infty}
$$

where $\mu_{\mathrm{o}}$ is the reference viscosity at low shear rates and $\mu_{\infty}$ is an asymptotic value of viscosity at large shear rates, $\dot{\gamma}=0.5 \sqrt{\mathrm{I}_{2}}$ with the second invariant $\mathrm{I}_{2}$ of the rate of strain tensor, $\mathrm{m}$ is a material index and $\lambda$ is a material constant. 
For generality, the following non-dimensionalisation is adopted

$$
\mathbf{x}^{*}=\frac{\mathbf{x}}{\mathrm{L}}, \mathbf{u}^{*}=\frac{\mathbf{u}}{\mathrm{V}}, \mu^{*}=\frac{\mu}{\mu_{\mathrm{c}}}
$$

where $\mathrm{L}, \mathrm{V}$ and $\mu_{\mathrm{c}}$ are characteristic length, velocity and viscosity respectively. We take $\mathrm{L}$ to be the diameter of the stirrer, $\mathrm{V}$ to be the speed of the vessel, $\mu_{\mathrm{c}}$ to be 1 Pa.s. This leads to a Reynolds number definition,

$$
\operatorname{Re}=\frac{\rho L V}{\mu_{c}}
$$

where $\rho$ is the fluid density. Hereforth, we discard the * notation for ease of presentation.

The algorithm used in this work follows references (Hawken et al., 1990, Ding et al., 1995 and Matallah et al., 1998). Briefly, a Taylor-Galerkin algorithm is employed to solve the governing equations relating to the conservation of mass and momentum. A time stepping scheme is derived through Taylor series expansions up to second order in time step and a twostep predictor-corrector scheme is assumed. This, in conjunction with a second-order pressure correction method to accommodate the incompressibility constraint, produces a fractionalstaged equation system to solve of three distinct phases over each time step. A semi-implicit treatment of a Crank-Nicolson type is adopted for diffusion terms. A Galerkin finite element spatial discretisation renders a fully-discrete system with the choice of piecewise continuous quadratics for velocity, and linears for pressure. The system is specified as

stage 1a:

$$
\left[\frac{2 \operatorname{Re}}{\Delta \mathrm{t}} \mathbf{M}+\frac{1}{2} \mathbf{S}_{\mathrm{u}}\right]\left(\mathbf{U}^{\mathrm{n}+\frac{1}{2}}-\mathbf{U}^{\mathrm{n}}\right)=\left\{-\left[\mathbf{S}_{\mathrm{u}} \mathbf{U}+\operatorname{Re} \mathbf{N}(\mathbf{U}) \mathbf{U}\right]+\mathbf{L}^{\mathrm{T}} \mathrm{P}\right\}^{\mathrm{n}}
$$

stage $1 b$ :

$$
\left[\frac{\operatorname{Re}}{\Delta \mathrm{t}} \mathbf{M}+\frac{1}{2} \mathbf{S}_{\mathrm{u}}\right]\left(\mathbf{U}^{*}-\mathbf{U}^{\mathrm{n}}\right)=\left[-\mathbf{S}_{\mathrm{u}} \mathbf{U}+\mathbf{L}^{\mathrm{T}} \mathrm{P}\right]^{\mathrm{n}}-\operatorname{Re}[\mathbf{N}(\mathbf{U}) \mathbf{U}]^{\mathrm{n}+\frac{1}{2}}
$$

stage 2:

$$
\mathbf{K}\left(\mathbf{P}^{\mathrm{n}+1}-\mathbf{P}^{\mathrm{n}}\right)=-\frac{2}{\Delta \mathrm{t}} \mathbf{L} \mathbf{U}^{*}
$$

stage 3:

$$
\frac{\operatorname{Re}}{\Delta \mathrm{t}} \mathbf{M}\left(\mathbf{U}^{\mathrm{n}+1}-\mathbf{U}^{*}\right)=\frac{1}{2} \mathbf{L}^{\mathrm{T}}\left(\mathbf{P}^{\mathrm{n}+1}-\mathbf{P}^{\mathrm{n}}\right)
$$


where $\mathbf{U}^{\mathrm{n}}, \mathbf{U}^{\mathrm{n}+1}, \mathbf{P}^{\mathrm{n}}$ and $\mathbf{P}^{\mathrm{n}+1}$ are nodal vectors of velocity and pressure at time $\mathrm{t}^{\mathrm{n}}$ and $\mathrm{t}^{\mathrm{n}+1}$

respectively; $\mathbf{U}^{*}$ is an intermediate nodal velocity vector introduced in step $1 \mathrm{~b} ; \mathbf{M}, \mathbf{S}_{\mathbf{u}}, \mathbf{N}(\mathbf{U})$, $\mathbf{K}$ and $\mathbf{L}$ are mass matrix, momentum diffusion matrix, convection matrix, pressure stiffness matrix and divergence/pressure gradient matrix, respectively. The details of the above matrices can be found in the literature, under the same references as for the algorithm.

\subsection{Problem Specification}

The geometries considered are essentially the same two basic geometries considered for the experimental case, both are cylindrical vessels one with one stirrer and the other with two. Again the fluid is driven by the outer vessel wall and base, while the lid at the top of the vessel and the rod(s) attached to it are fixed, see Figure 1.

Tetrahedral elements are employed in the current work. To create a three-dimensional finite element mesh, first each brick element is formed, which is then divided into six tetrahedra. The height of the vessel is divided into five uniform layers. For the vessel with one stirrer, the horizontal plane is divided into 120 quadrilaterals. This leads to 3,600 tetrahedral elements, 5,720 velocity nodes and 840 pressure nodes, resulting in total in 18,000 degrees of freedom. For the vessel with two stirrers, the horizontal plane is divided into 280 quadrilaterals. Correspondingly, this leads to 8,400 tetrahedral elements, 13,145 velocity nodes and 1,902 pressure nodes, and a total of 41,337 degrees of freedom.

In order to parallel the experimental work (see section 4.1 below) two types of fluid models are incorporated in this work, a Newtonian model and an inelastic model with shear rate dependent viscosity as described in $\mathrm{Eq}(1)$, where $\mu_{\mathrm{o}}=1.05$ Pa.s, $\mu_{\infty}=0.001$ Pa.s, $\lambda=$ $0.083 \mathrm{~s}$ and $\mathrm{m}=0.62$. These models are chosen to have rheological properties, which match those of the model fluids used in the experimental programme.

\section{$4 \quad$ Results and Discussion}

\subsection{Model fluids}

Figure 2 shows shear viscosity data obtained with a controlled stress rheometer, a TA instruments AR1000, for the simplified dough itself, watered dough and dilute CarboxyMethyl-Cellulose CMC solutions. It can be seen that the CMC solutions, though considerably less viscous than the dough have similar shear-thinning characteristics over the measured range of shear rates and have very similar viscosity profiles to the watered dough. Note that 
the maximum shear rates measured with this rheometer are approximately the same as the maximum shear rates encountered in the model geometries, which indicates the relevance of the measured range.

Figure 3 shows corresponding dynamic data, storage modulus $G^{\prime}$ and loss modulus G', for these materials. Again the CMC exhibits similar trends to the dough, though the magnitudes of its dynamic modulii are considerably lower. It can be inferred from the graph that the dough itself is more elastic than viscous ( $G^{\prime}>G^{\prime}$ '), while for the most dilute CMC solution the converse is true. However, the higher concentration CMC's and the watered dough have comparable levels of viscoelasticity.

Figures $4 \mathrm{a}$ and $4 \mathrm{~b}$ present additional shear viscosity data, for the dough and the $4 \%$ CMC solution, obtained with a capillary rheometer. This is compared with dynamic data from the AR1000 via the CoxMertz rule (Cox and Mertz 1958). By considering flow through an orifice and applying contraction flow analysis (Binding, 1988), extensional viscosities are also obtained. The significant result here is that the extensional viscosity of the CMC shows a similar level of tension thinning to the dough and the Trouton ratios of both the dough and the CMC are noticeably above the theoretical Newtonian value of 3 (Trouton, 1906).

Although various other model fluids were also investigated the $1 \% \mathrm{CMC}$ solution was found to be the most suitable candidate for the inelastic case. While a glucose syrup and water solution with viscosity matching the first Newtonian level of the CMC (i.e. 0.5Pas) is used for the Newtonian case.

The format followed for the remainder of this section, will be to present experimental data obtained using the techniques outlined in section 2 and to compare each set of data with the relevant numerical simulations. Results for both the Newtonian and inelastic solutions are found to be virtually identical in terms of streamlines and velocity vectors (see section 4.3 for an explanation) : for these cases, only data for the inelastic fluid are shown.

\subsection{Streamlines}

Stills photographs for the two basic geometries have been obtained for both the syrup and CMC solutions at various speeds/Reynolds numbers. Figures 5 and 6 provide an indication of the streamline patterns produced by the CMC $1 \%$ solution. Since the flow and hence the particles are assumed to move along these streamlines. In fact, visual observation of the particles in the flow showed that very few particles entered or left the plane of laser light, demonstrating that there is little flow in the axial direction. For the one-rod case, a central 
vortex evolves opposite to the stirrer. This is almost symmetrical in form at the lowest speed ( $25 \mathrm{rpm}$, equivalent to $\operatorname{Re}=2$ ). The pattern of the flow is seen to twist counter-clockwise away from the horizontal central axis, as the counter-clockwise rotation speed is increased, due to the increasing influence of fluid inertia. With the two-rod geometry a pair of vortices emerge, generated by the separating flow around each stirrer. Again the flow pattern is seen to distort anti-clockwise due to increasing speed/inertia.

Numerical simulations for the two geometries have been conducted for both Newtonian and inelastic materials at various speeds. The stream function patterns derived for the inelastic material, at Reynolds numbers equivalent to the experimental case, are displayed in Figures 7 and 8. It can be seen that these results agree qualitatively well with the counterpart experimental visualizations. The plots are taken in a plane at three-fifths height of the vessel and the value of the stream function is assumed to be zero at the outer vessel wall. Ten contour levels are taken for the stream function between values of zero and one, thereafter adopting an interval spacing of 0.05 for the vessel with one stirrer and 0.0233 for the vessel with two stirrers. For the one-stirrer case, as with the experiments, a central vortex appears horizontally opposite to the stirrer, which is symmetrical at the lowest speed (equivalent to $\operatorname{Re}=2$ ). It is observed that the core value of the streamlines declines as the speed increases, noted through the maximum value reported. Again, as with the experiments, the pattern of the flow is seen to contort and twist in an asymmetrical fashion, away from the horizontal central axis, as the speed of rotation is increased. With a two-stirrer configuration, similar comments apply on asymmetry with increasing speed. Here, again as seen in the photographs, two vortex centres emerge driven by the separating flow around each stirrer. The results for a Newtonian fluid bear close resemblance to the above. The only feature worthy of note is that vortex intensities are slightly larger for Newtonian fluids.

\subsection{Vortex Centres}

It is also desired to obtain a measure of the agreement between the experimental laser speckle streamlines and the numerical stream functions as a function of depth in the geometry, in order to investigate the three-dimensional nature of the flow. To this end the positions of the vortex centres are determined for speeds corresponding to the Reynolds numbers of 2, 4, 8 and16 and at the heights $\mathrm{z} 1, \mathrm{z} 2, \mathrm{z} 3$ and $\mathrm{z} 4$. The coordinates of the centres are then plotted in conjunction with simulation data for the same cases in Figures 9 for the one-rod geometry and Figure 10 for the two-rod geometry \{dropped ref to $9 \mathrm{~b}$ and $10 \mathrm{~b}$ \}. The 
precise location of vortex centre is a subjective issue, estimated by eye, a procedure itself subject to experimental error in quantification.

As for the laser speckle photographs the experimental data show that, as the speed of rotation increases, the vortex centres rotate counter-clockwise i.e., with the direction of rotation of the vessel. This is true for all four heights. However, with decreasing height the centres are located closer to the centre of the geometry, for both geometries. In fact, for the two-rod case at the lowest height z1 and fastest speed (equivalent to Reynolds number 16), the two vortex centres merge together to form a single vortex. This convergence is due to the influence of the rotating base of the vessel at this proximity and can also be observed at $\mathrm{z} 2$ for higher speeds. Thus, for the two-rod case there are two distinct vortices which come closer together on moving down the vessel and merge into one near the base. Compatible results for the Syrup solution are difficult to distinguish from those of the CMC. We note that CMC itself, may reflect some elastic properties and hence may constitute a source of some departure from inelastic simulations.

The vortex centres generated by the numerical simulations are located in the same areas of the geometry cross-section as those from the experiments, see Figures 9 and 10. The predicted streamlines follow the experimental trends: moving counter-clockwise with increasing Reynolds number and shifting closer to the geometry centre with decreasing height. In the two-rod instance, again convergence of the twin vortices is noted at the highest speed $(\operatorname{Re}=16)$ and at the lowest plane $(\mathrm{z} 1)$, close to the vessel-bottom. For the single-rod, the oval vortex shape of lower speeds transforms to a broad-bean shape at the highest speed, this being more prominent at the higher plane $(\mathrm{z} 4)$, close to the vessel-lid (also noted in the experiments). The simulated vortex centres coincide closely with experimental results at the centre plane. On other planes, some departure is noted as one moves away from the centre plane. Precision on vortex centre determination is elusive, both experimentally and computationally. Discrepancies in modelling the flow are bound to arise towards the ends of the vessel due to the approximations necessary to model the contact-point problems specifically, between rod-vessel bottom and vessel side-top-plate. In addition, we point out that mesh refinement in the depth-wise direction is fairly coarse for pragmatic reasons alone. Taking these issues into account, the agreement between simulations and experiments is reasonable (or gratifying).

\subsection{Velocity Vectors - High Speed Camera}


Vector plots obtained using the high-speed camera technique for the one and two-rod geometries with the $1 \% \mathrm{CMC}$ solution are shown in figures 11 and 12, respectively. Due to the way the vectors are calculated, from the motions of individual tracer particles along their streamlines, the length of the vectors displayed is directly proportional to their magnitude and every vector starts and finishes on a streamline. Although the velocity vectors are estimated, provided the curvature of the streamlines is not too great, precision will lie within a few percent (i.e., $<3 \%$ ) of the actual velocities. The combination of velocity vectors and their associated streamlines is most useful, as it contains information on the direction and the relative strength of the flow. Clearly, the streamlines reflect the same patterns as the laser speckle photographs for the corresponding Reynolds number of 2. Unfortunately, at present, higher Reynolds number cannot be studied using this technique as insufficient light is afforded by the lasers to accommodate higher shutter speeds. It is envisaged that the future acquisition of a light-intensifying lens will overcome this difficulty. The velocity vectors themselves are, as expected, a maximum (typically $0.25 \mathrm{~ms}^{-1}$ ) near the outer moving wall and fall away appreciably on moving inwards towards the vortex centre(s). By considering vectors that lie approximately parallel to each other, on adjacent streamlines, local shear-rates can also be estimated from the velocity gradients. Hence, assuming that the fluid velocity is zero adjacent to the stationary rods (i.e., a no-slip condition holds), it can be inferred that the maximum shear-rates will occur near the outsides of the rods. Virtually identical results are observed for the Newtonian Syrup solution. The reasons for this are that, at $1 \%$ concentration, the CMC is only slightly elastic and the difference in shear viscosity to that of the Newtonian fluid (due to shear-thinning), only becomes significant at the highest shear-rates encountered in the geometry.

Numerical data for the equivalent geometries and model fluids are shown in figures 13(a) and (b) and 14(a) and (b). Here, results on the single central z2 plane are presented, the (a) figures show typical solution contours of shear-rate and the (b) figures contours of the localized rate-of-work done on the fluid. Filled circles are used to indicate the positions where the maximum values occur and the range of values represented by the contours are given in the figure legend. For both the one and two-rod geometries maximum values of shear-rate and rate-of-work done are observed in the narrow region between the vessel and the stirrer. Since the stirrer is stationary and the vessel is rotating, velocity gradients peak around the stirrer, particularly in the constricted gap section between the stirrer and the vessel wall. Hence, more shearing and stretching of fluid occurs in this region and so the rate-of-work done peaks to a 
maximum there. Thus, there is basic agreement between the experimental result, that the highest velocity gradients are observed just outside the rods, and the numerical result, that the maximum shear-rates and rates-of-work done also occur there. Note that, the evaluation of the rate-of-work done by the model is a critical component of the overall study. We remind that it is desired to design mixing geometries, which do the maximum amount of useful work on the dough. In Figure 15 (a), three-dimensional plots of pressure, shear-rate, extension-rate and rate-of-work done for the two-rod mixer are included at speed $\mathrm{Re}=8$ to convey the variations of these variables in depth. Aside from above comments, deformation rates peak at the lid, extension is noticeable at the base near the rods, rate-of-work is also prominent near the lid on the narrow-gap side of the stirrers. Extension-rate in three-dimensional view varying with increase of speed is given in Figure 15 (b). Here, greatest extension corresponds to the lidregion (with the vessel motion), and as speed increases, there is wider influence around the lid away from the stirrers.

\subsection{Velocity Vectors - Laser Doppler}

Laser Doppler data for the geometries, both one and two-rod, with the outer vessel fixed and the inner rods rotating with $1 \% \mathrm{CMC}$ solution are shown in figure 16 . Unlike the pervious data obtained with the analysis of the high-speed camera images, the velocity vectors here are located on a fixed axial grid, as described in section 2.2. As a result, it is more difficult to gain an impression of the flow fields, since these vectors are not associated with the same streamlines. Nevertheless, it is still possible to detect a small re-circulation near the inside of the rod of the one stirrer geometry and an elongated oval-shaped flow between the rods of the two-stirrer geometry. For both geometries, the maximum velocity vectors $\left(0.25 \mathrm{~ms}^{-}\right.$ ${ }^{1}$ ) occur in the wake of the rotating rods, falling off to minima near the stationary walls and close to the geometry centre. They will be even higher adjacent to the rods, but due to the scale of the grid, cannot be measured at this proximity. Again, as for the simpler geometries with the outer vessel rotating, the maximum shear-rates will be situated between the rods and the vessel wall. Prakash [1996] and Prakash and Kokini [1999] have also performed studies using LDA to determine the velocity vectors associated with the flows in model mixers. They used the measured velocities to estimated velocity gradient values, and from these data sets, determined shear-rate distributions in their vessels.

Numerical simulations for the more advanced geometries are shown in figure 17. These vector plots are obtained by adding the rotation back in to the appropriate solutions for the fixed-rod cases. This is achieved by translating the motion of the vessel to that of the field. 
In other words, by subtracting the product $\mathrm{r} \Omega$ from the velocity field obtained from the vessel rotating case. This would make the vessel stationary and the stirrers rotate in the opposite direction, i.e. clockwise. Although the velocity vectors shown here are positioned on a different grid and use a different scale to represent magnitude than those of the laser Doppler results, they do exhibit the same flow patterns and trends as the experimental data. That is, the re-circulations occur in the same places and the largest velocities occur in the proximity of the stirring rods. This is an encouraging result as these geometries represent a more realistic mixing regime than the simplified cases considered earlier. It illustrates the progress of the model towards the ultimate aim of simulating the mixing of commercial dough within industrial mixers.

\section{Conclusions}

Due to the fact that flow fields in the simplified geometries under investigation have only a limited axial component and are steady, it has proven possible to use the conventional laser scatter technique with stills photography to obtain a clear picture of the pattern of streamlines associated with the model fluids. Excellent agreement with experiments is observed in stream function generated by the numerical simulation for both the Newtonian and shear thinning fluids in both the one and two-rod geometries. On vortex centres at several heights through the vessel, qualitatively there is corroboration between simulation and experiment.

With the incorporation of a modern digital high-speed camera system and careful data analysis procedure, it is possible to enhance the laser scatter data by producing the velocity vectors associated with these streamlines. Further, as the velocity vectors are located on the streamlines, the data are far more instructive than those obtained with a laser Doppler technique, where velocity vectors are presented on a fixed grid. The distribution of velocities in the geometries indicates that the largest shear-rates occur just outside the stirring rods. This is in full agreement with the numerical result, where the maximum shear-rates are located next to the outside of the rods. In addition the rate-of-work done is also shown to be a maximum at this position. This is an important parameter in assessing the overall efficiency of a mixing process.

Laser Doppler Anemometry has been incorporated in assessing the velocity vectors associated with the flow fields of more realistic geometries where the rods are made to rotate and the vessel is fixed. This is a more complex flow to analyse numerically, since any mesh 
used must be made to rotate with the rods. Good agreement between the flow fields and the relative magnitudes of the velocity vectors are obtained between the experimental and numerical data. The fact that the two sets of data correlate provides the strongest evidence that the model is progressing successfully.

To conclude, the principal aim of the study, to develop numerical simulations of simplified filled dough mixing scenarios and to verify this work experimentally, has been successfully accomplished. We have demonstrated the use of a numerical inelastic flow solver as a prediction tool for this industrial flow problem and been able to provide physically realistic simulations for this complex mixing process using inelastic model fluids.

Promising future directions of this work are as follows. We are addressing the transient free surface problem and the phenomena of wetting-and-peeling boundary contact, which arises in the dough mixing process. Currently more complicated material properties such as viscoelasticity are being investigated, accounting for memory and variable extensional properties. Through the predictive capability generated, we shall be able to apportion power input and relate this to mixer design that will ultimately impact upon the processing of dough products.

\section{Acknowledgements}

We wish to acknowledge Dr. D. Ding for his technical assistance in this work and the following collaborating bodies for their support: BBSRC for providing the principle funding of the project; RHM technology, United Biscuits Ltd., Pillsbury Enc. and Mono Equipment for their additional financial contributions and technical guidance; RHM for providing the model mixer and EPSRC (EBSRC?) equipment pool for supplying the Laser Doppler Anemometer.

\section{References}

Bicen, A.F. (1982). Refraction correction for LDA measurements in flows with curved optical boundaries. TSI Quarterly, 5 (2), 10-12.

Binding, D. M. (1988). An approximate analysis for contraction and converging flows. $J$. Non-Newtonian Fluid Mech., 27 (2), 173-189.

Boger, D.V. (1982). Circular entry flows of inelastic and viscoelastic fluids. Advances in Transport Processes, Wiley Eastern Ltd., 43-104.

Cochrane, T., Walters, K., Webster, M.F. (1981). On Newtonian and non-Newtonian flow in complex geometries. Philos. Trans. Roy. Soc., A301, 163-181. 
Couch, M.A., Binding, D.M. (2000). Experimental Simulation of Dough Kneading. Rheology 2000 - XIII Int. Congress on Rheology, BSR, Glasgow, 2, 351-353.

Cox, W.P., Mertz, E.H. (1958). Correlation of dynamic and steady flow viscosities. J. Polym. Sci., 28, 619-622.

Ding, D., Townsend, P., and Webster, M. F. (1995). On computations of two and threedimensional unsteady thermal non-Newtonian flows. Int. J. Num. Meth. Heat Fluid Flow 5, 495-510.

Ding, D., Webster, M.F. (2000). Three-dimensional Numerical Simulation of Dough

Kneading. Rheology 2000 - XIII Int. Congress on Rheology”, BSR, Glasgow, 2, 318-20.

Evans, R.E., Walters, K.W. (1985). Flow characteristics associated with abrupt changes in geometry in the case of highly elastic liquids. J. Non-Newtonian Fluid Mech., 20, 11-29.

Hawken, D. M., Tamaddon-Jahromi, H. R., Townsend, P., and Webster, M.F. (1990). A Taylor-Galerkin-based algorithm for viscous incompressible flow. Int. J. Num. Meth. Fluids, 10 (3), 327-351.

Matallah, H., Townsend, P. and Webster, M. F. (1998). Recovery and stress-splitting schemes for viscoelastic flows. J. Non-Newtonian Fluid Mech., 75, 139-166.

Prakash, S. (1996). Characterisation of shear rate distribution in a model mixer using LaserDoppler Anemometry. Ph.D. Dissertation, Rutgers, The State University of New Jersey.

Prakash, S., Kokini J.K. (1999). Estimation and prediction of shear rate distribution in a model mixer. Journal of Food Processing, 44, 135-148.

Trouton, F. T. (1906). Proc. Roy. Soc., A77, 426.

\section{APPENDIX}

\section{FIGURE LEGENDS}

Figure $0 \quad$ Model mixer, in operation, and baked product.

Figure 1 Schematic diagram of the filled model geometry for the one-rod geometry (the two-rod case is represented by the dashed outline.

Figure 2 Shear viscosity versus shear rate data for an idealised industrial dough, watered doughs (note the percentages represent the water content relative to the idealised dough at $100 \%$ ) and dilute CMC solutions.

Figure 3 Loss and Storage modulus data versus frequency for an idealised industrial dough, watered doughs and dilute CMC solutions. 
Figure 4(a) Shear and extensional viscosity data versus strain rate for an idealised industrial dough.

Figure 4(b) Shear and extensional viscosity data versus strain rate for a 4\% CMC solution.

Figure 5 Laser speckle photographs for $1 \% \mathrm{CMC}$ solution in the one-rod geometry at various Reynolds numbers.

Figure 6 Laser speckle photographs for $1 \% \mathrm{CMC}$ solution in the two-rod geometry at various Reynolds numbers.

Figure 7 Stream functions for the inelastic material in the one-rod geometry at various Reynolds numbers.

Figure 8 Stream functions for the inelastic material in the two-rod geometry at various Reynolds numbers.

Figure 9 Co-ordinates of the vortex centres for 1\% CMC solution (experimental) and the inelastic material (numerical) in the one-rod geometry at various Reynolds numbers and depths.

Figure 10 Co-ordinates of the vortex centres for 1\% CMC solution (experimental) and the inelastic material (numerical) in the two-rod geometry at various Reynolds numbers and depths.

Figure 11 High-speed camera - velocity vector data for $1 \% \mathrm{CMC}$ solution in the one-rod geometry at Reynolds number 2.

Figure 12 High-speed camera - velocity vector data for $1 \% \mathrm{CMC}$ solution in the two-rod geometry at Reynolds number 2.

Figure 13 Contours of shear rate and rate of work done for the inelastic material in onerod geometry at Reynolds number 2 .

Figure 14 Contours of shear rate and rate of work done for the inelastic material in the two-rod geometry at Reynolds number 2.

Figure 15(a) Various field variables throughout the two-rod vessel, $\mathrm{Re}=8$.

Figure 15(b) Extension-rate patterns throughout the two-rod vessel, increasing speed.

Figure 16 Laser Doppler Anemometer - velocity vector data for 1\% CMC solution in the one and two rod geometries at Reynolds number 2.

Figure 17 Velocity vectors for the inelastic material in the one and two rod geometries at Reynolds number 2. 

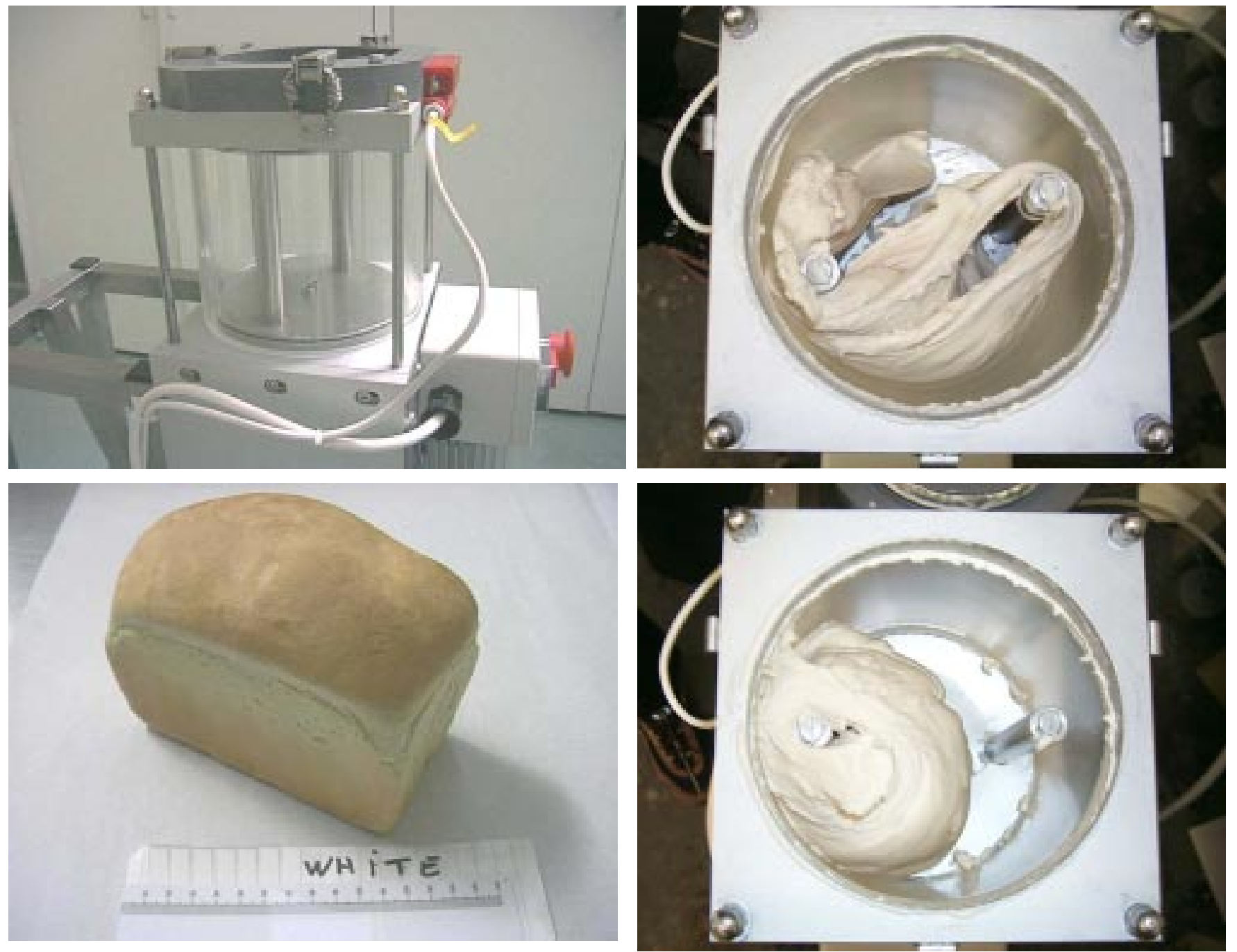

Figure 0. Model mixer, in operation, and baked product. 


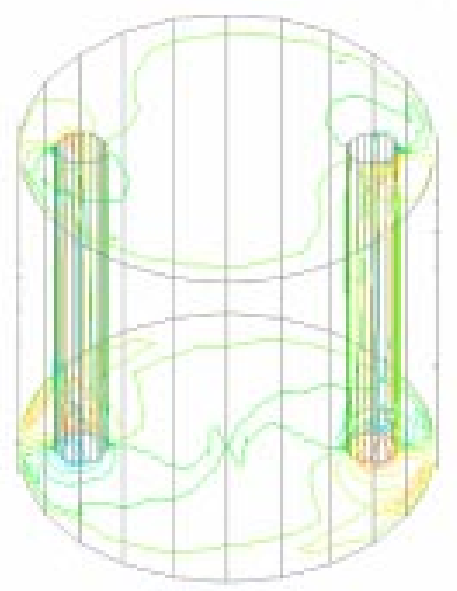

Pressure

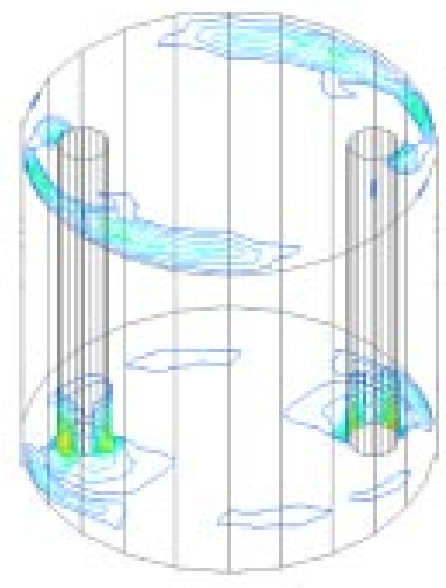

Extension-rate

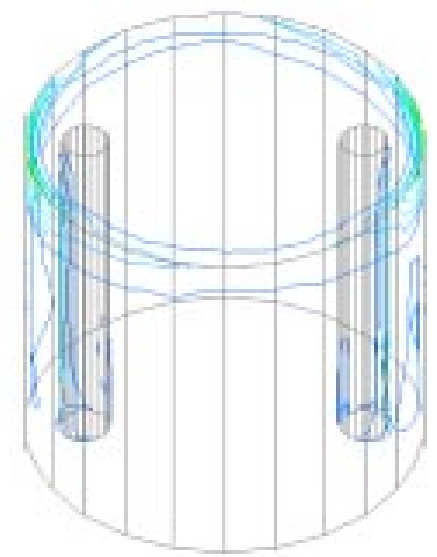

Shear-rate

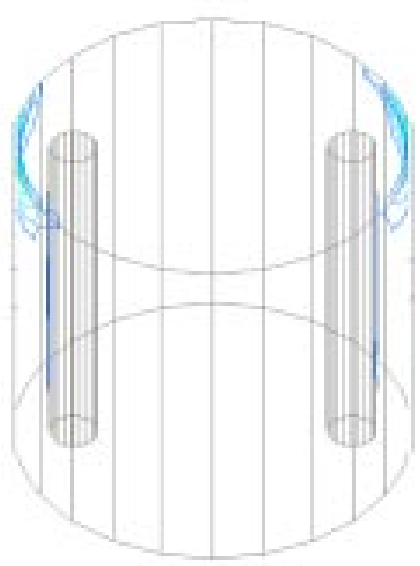

Rate-of-work done

Figure 15 (a) Various field variables throughout the two-rod vessel, $R e=8$. 

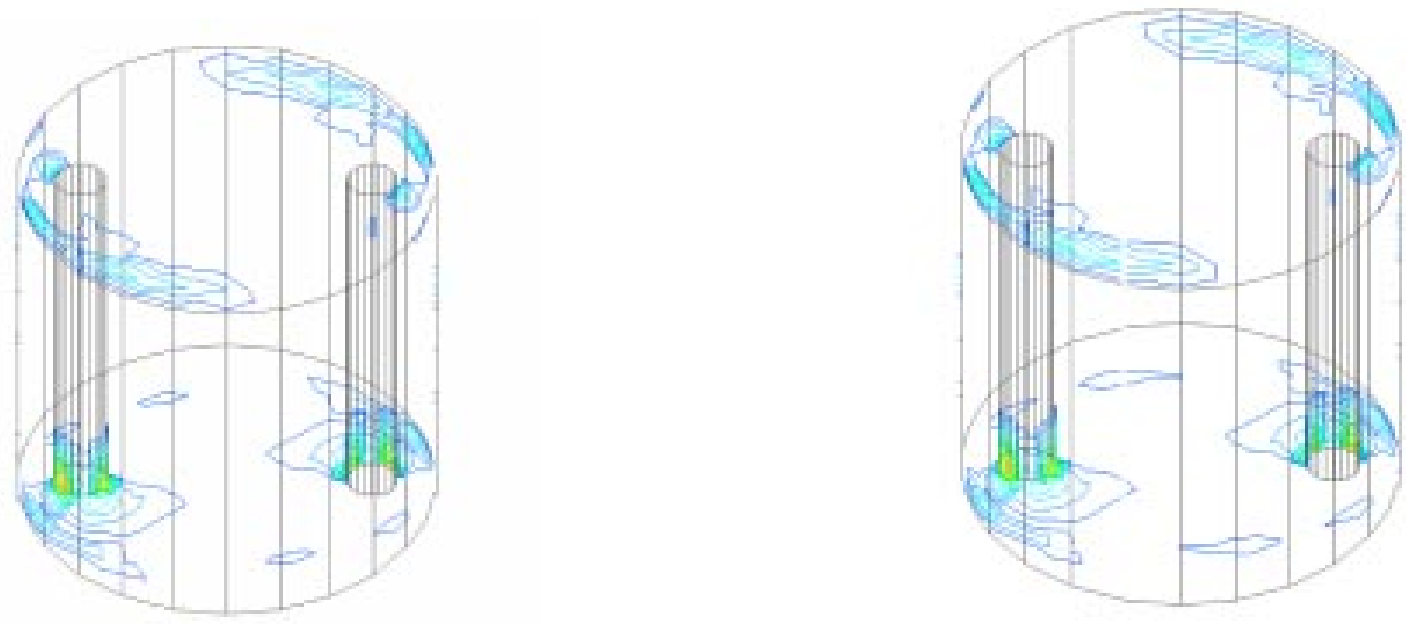

\section{$\mathrm{Re}=4$}

\section{$\mathrm{Re}=2$}
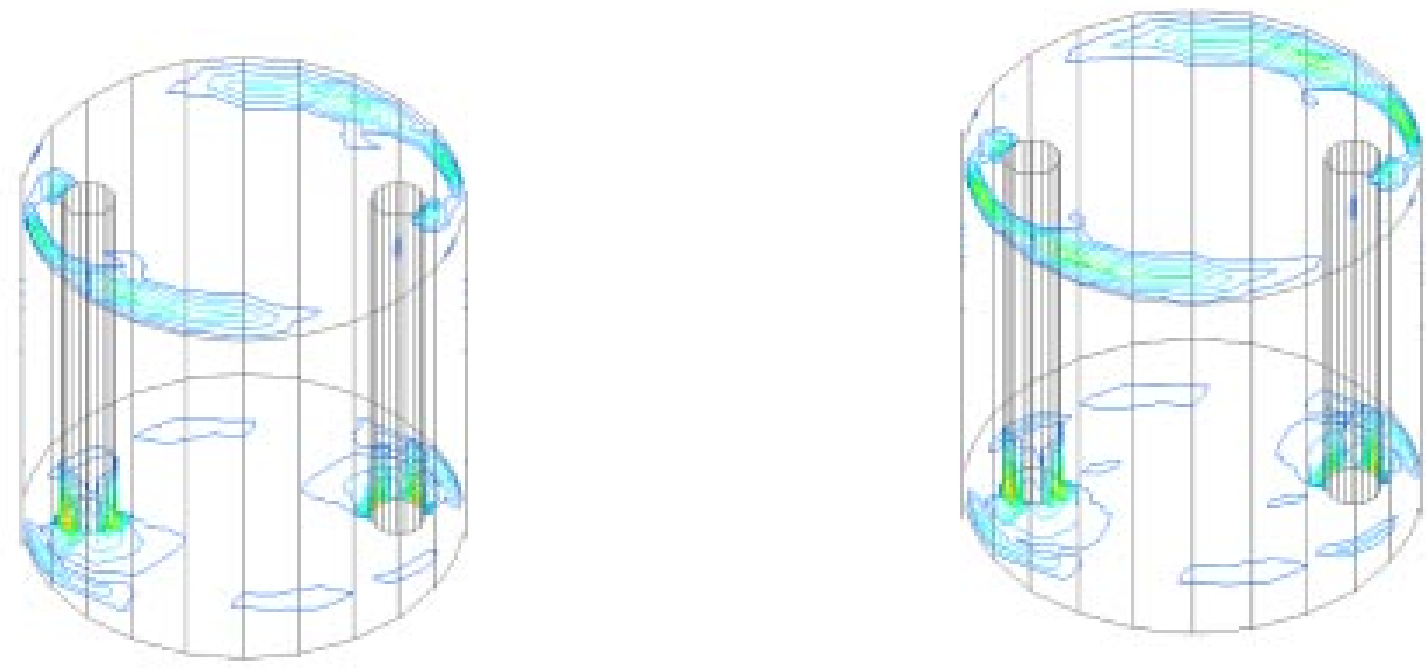

$\mathrm{Re}=16$

\section{$\mathrm{Re}=8$}

Figure 15 (b) Extension-rate patterns throughout the two-rod vessel, increasing speed. 